Gazi University
Journal of Science
$\mathrm{http} / /$ dergipark.gov.tr/gujs

\title{
Comparison Study on Economic Load Dispatch Using Metaheuristic Algorithm
}

\author{
Widi ARIBOWO* \\ Universitas Negeri Surabaya, Faculty of Engineering, 60231 Surabaya, Jawa Timur, Indonesia
}

\author{
Highlights \\ - This paper focuses on comparison study on Economic Load Dispatch between Metaheuristic algorithm. \\ - This paper compares 6 metaheuristic methods. \\ - STSA is slightly superior.
}

\begin{tabular}{|c|}
\hline Article Info \\
\hline $\begin{array}{l}\text { Received: } 04 \text { Nov } 2020 \\
\text { Accepted: } 17 \text { Mar } 2021\end{array}$ \\
\hline Keywords \\
\hline $\begin{array}{l}\text { Economic load dispatch } \\
\text { Metaheuristic } \\
\text { Optimization } \\
\text { Nature inspired } \\
\text { algorithm }\end{array}$ \\
\hline
\end{tabular}

\begin{abstract}
This paper presents an approach to overcome economic load dispatch (ELD) using a metaheuristic algorithm. Economic load dispatch (ELD) is one of the most important problems in a power system, and solving it quickly is extremely important. The main problem that will be addressed in this paper is how to optimize the economy of the power grid with various operational limitations, the loss in transmission line power, and consider minimizing the fuel costs produced. In this study, some of the newest metaheuristics inspired by nature will be explored, namely Seagull Optimization Algorithm (SOA), Marine Predator Algorithm (MPA), Sine Tree-Seed Algorithm (STSA), Chimp Optimization Algorithm (ChOA), Equilibrium Optimizer (EO), and Giza Pyramids Construction (GPC). The performance appraisal of the method applied in this study was tested using 2 case studies, namely a system with 3 and 6 power system units. The results are presented by comparing between metaheuristic and mathematical methods. The experimental results is showed that the Sine Tree-Seed Algorithm (STSA) is presented the best performance with various case studies with constraints.
\end{abstract}

\section{INTRODUCTION}

Electric power is a very important requirement in the modern era 4.0. The increasing demand for electric power causes the electric power that must be supplied by the generator to be very large. Renewable energy sources are a determining factor for industrial development that can improve people's living standards. In addition, technological advances and developments have also contributed greatly to the increasing demand for electricity. The increase in electrical energy from the consumption side will be a constraint on the generation side [1].

Planning, managing the generation, and distribution of electrical energy are required to meet the demands of consumers with the aim of increasing the quantity and quality of energy produced. Improving the quality of electrical energy is also very influential in increasing the efficiency and reliability of the system. Optimization of generator scheduling in electric power systems is necessary because the generation and distribution processes in the electric power system require very large costs. Coordination between generators is needed in an effort to optimize the scheduling generator to obtain minimum costs.

Each generator has its own characteristics. The difference in the characteristics of the generating unit causes each unit generators to have different portions in supplying the load of an electric power system. The operating cost of an electric power system is the largest cost of operating an electric company. In electric power system operation, fuel costs occupy the largest cost, namely $80 \%$ of the overall operating costs. 
Economic Load Dispatch (ELD) is a calculation analysis to obtain a calculation of the minimum cost of a generation which is indispensable to minimize and optimize expenses [2]. ELD is one of the fundamental issues in the operation of the electric power system [3]. Various methods have been presented in solving ELD problems using optimization techniques and programming methods based on mathematical methods. Various methods are often referred to as conventional methods. These include the baseline and participation factor methods, lambda, and gradients. The conventional method becomes a problem when dealing with systems that have nonlinear characteristics. The nonlinear characteristics of a generator include valve-point loading effects or multi-fuel source options for generating units, ramp-rate limitations, prohibited operating zones, and cluttered cost function.

The development of artificial intelligence methods in several decades encourages solving a problem using artificial intelligence computing, including optimization in ELD. Several studies have been presented and have performed well in solving ELD problems. Several artificial intelligence methods particularly the metaheuristic method have been used in solving ED problems in the past 5 years such as the chaotic bat algorithm (CBA) method which is a variant of the basic bat algorithm by entering chaotic sequences to improve its performance [4-7], Artificial Bee Colony Algorithm (ABC) which mimics the intelligent honeybee foraging behavior [8-10], grey wolf optimizer (GWO) inspired by hunting rules wolves and the grey wolf social hierarchy [11-14], and cuckoo search algorithm inspired by the interesting breeding behavior of cuckoo [15-18].

This paper will explore the potential of six metaheuristic methods, namely, Seagull Optimization Algorithm (SOA) [18], marine predator algorithm (MPA) [19], Sine Tree-Seed Algorithm (STSA) [20], Chimp Optimization Algorithm (ChOA) [21], Equilibrium Optimizer (EO) [22], and Giza Pyramids Construction (GPC) [23] in solving ELD problems. The test is using 2 different case studies and is based on several constraints. Testing is using 3 and 6 units of the power system. Several studies on ELD using metaheuristic methods have been reported, but there is still a lot of room to be explored to find the best solution in solving ELD. The contribution of this paper is

1. The application of the latest and promising metaheuristic methods in solving ELD problems with constraints. On the other hand, it is presenting the metaheuristic behavior used.

2. In-depth analysis of the metaheuristic methods used in this paper including 23 benchmarks.

3. Tested the method used in 2 case studies and compared between algorithms

The paper is organized as follows: the second part provides a complete study of ELD and a brief description of the metaheuristic methods used in this paper. Section 3 presents the results and performance comparison analysis of the metaheuristic method. In the last section, a conclusion is drawn.

\section{MATERIAL METHOD}

\subsection{Economic Load Dispatch}

Problems that often arise in a power system are fluctuating every certain time period. To supply loads economically, the economic load dispatch (ELD) calculation is carried out for each of the large loads. Economic load dispatch (ELD) is the big issue that must be resolved in the operation of the electric power system. Economic load dispatch is defined as the process of allocating generating power to a generator so that the system at load can be supplied economically. In general, the cost function of each generating unit can be formulated mathematically as an objective function, as given in the equation:

$$
M_{t}=\sum_{i=1}^{n} M_{i}\left(D_{i}\right)
$$

The cost in Equation (1) can be derived in quadratic form as in Equation (2) for minimization purposes [24].

$$
M_{i}\left(D_{i}\right)=a_{j} D_{i}{ }^{2}+b_{j} D_{i}+c_{j},
$$


where $D_{t}$ is the total cost in $R / h$. $i$ is the sum of the generators, $D_{i}$ is the power of the $i$ th generator expressed in MW. $M_{i}\left(D_{i}\right)$ is the cost of generating $D_{i} . n$ is total number of generators in the power system. $a_{j}, b_{j}$ and $c_{j}$ are the cost coefficients of the $j$ th generator. There are several things that need to be considered when conducting economic dispatch. one of which is the load request. The total power generated by a generator must equal the load demand plus losses:

$$
\begin{aligned}
& \sum_{i=1}^{n} D_{i}=D_{D}+D_{L}, \\
& D_{L}=\sum_{j=1}^{n} \sum_{k=1}^{n} D_{j} B_{j k} D_{k},
\end{aligned}
$$

where $D_{j}$ and $D$ are the real power generations at the $j$ th and $k$ th buses. $B_{j k}$ is the loss coefficient that is constant under certain conditions. The power generated by the generator must be between its rating (Pmin and Pmax). The limits for each generator can be written:

$$
D_{i}^{\min } \leq D_{i} \leq D_{i}^{\max }(i=1, \ldots \ldots n) \text {, }
$$

where $D_{i}{ }^{\text {min }}$ is the lowest limit of the $i$ th generator output power. $D_{i}{ }^{\max }$ is the maximum output power of the $i$ th generator. The cost function is required an estimate of the optimal power unit value while minimizing objective criteria $\left(O_{c}\right)$,

$$
O_{c}=\sum_{i=1}^{n} M_{i}\left(D_{i}\right)+1000 \times a b s\left(\sum_{i=1}^{n} M_{i}\left(D_{i}\right)-D_{D}-\sum_{j=1}^{n} \sum_{k=1}^{n} P_{j} B_{j k} P_{k}\right) .
$$

\subsection{A Seagull Optimization Algorithm (SOA)}

The Seagull Optimization Algorithm is a metaheuristic algorithm that duplicates the life of gulls. Seagulls have a group habitat. Seagulls are omnivorous because they eat reptiles, earthworms, insects, fish etc. Seagulls are intelligent animals. This is helping the seagull to hunt for prey. Seagulls have migratory and hunting behavior. Seagulls are migrated in search of a new abundant source of food. This can be explained as follows:

- To avoid collisions between seagulls when migrating in groups, the seagulls will form a formation

- The seagulls that have the best survival will have followers

- The strongest seagull will be a reference in improving the initial position in migration

The SOA method generally has two exploration and exploitation processes.

\section{Migration (exploration)}

In the migration process, the seagulls will move from one place to another. The motion of seagull can be seen in Figure 1. In this process, several criteria must be met, namely:

- Reduce crashes

The reference value is used to calculate the position of the new search agent. This is to avoid collisions between seagulls

- Following the best seagull direction

In the first phase which has the aim of avoiding collisions between individuals. In this second phase, the seagull will follow the best individual.

- Stay tight to the best seagull.

The last phase of migration is for the seagulls to form by getting closer to the best seagull.

\section{Attacking (exploitation)}

In the exploitation process, the seagulls have a spiral motion when attacking their prey. They use their weight and wings. It is used as a variation when attacking and migrating. 


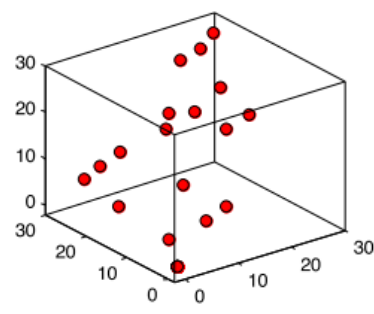

a)

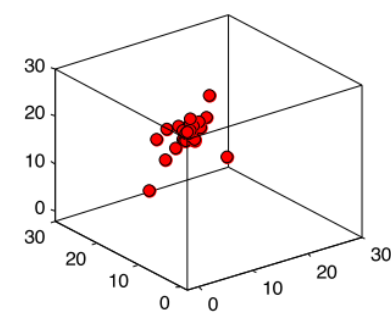

b)

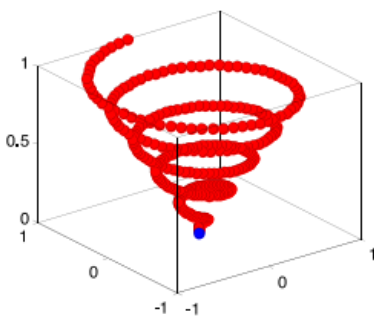

c)

Figure 1. The basic movement of seagull a) Reduce Crashes Formation, $b$ ) The Phase of Following the best seagull direction and c) Stay tight to the best seagull [18]

\subsection{Marine Predators Algorithm (MPA)}

The marine predator algorithm (MPA) is a new algorithm that is inspired by the behavior of predators and prey patterns in the sea. MPA has algorithms that naturally manage search strategies and optimal ranking policies between predators and prey in marine ecosystems. These creatures usually seek food continuously. Search symbols are represented by prey and predators. This is due to predators looking for prey. On the other hand, the prey is looking for food. The MPA algorithm has three main stages in optimizing by considering the speed ratio and the duplication of constituent elements. The phase in the MPA can be illustrated in Figure 2.

Phase 1: High-Speed Value ( $\mathrm{v} \geq 10)$

The predator will wait and watch the movement of the prey. Meanwhile, prey will explore the area in search of food. This phase occurs early. Similar to other metaheuristic methods, MPA is population-based. In phase 1, a mathematical model can be as follows:

$$
\begin{aligned}
& \overrightarrow{{S s_{l}}_{l}}=\overrightarrow{X_{b}} \otimes\left({\overrightarrow{\text { Elte }_{i}}}_{-} \overrightarrow{X_{b}} \overrightarrow{\otimes \text { Prey }_{i}}\right) i=1,2 \ldots . n \\
& {\overrightarrow{\text { Prey }_{i}}}=\overrightarrow{\text { Prey }}_{i}+C \times \overrightarrow{\text { rand }} \otimes \overrightarrow{S_{l}}
\end{aligned}
$$

where $\overrightarrow{S s_{l}}$ is the step size or the velocity of movement. The $\overrightarrow{X_{b}}$ notation is a vector containing random numbers following the normal distribution that represents the movements of the brownian. $\overrightarrow{r a n d}$ is a uniformly conditioned random number vector with values ranging from 0 to 1 and $C=0.5$ is a constant number.

Phase 2: Unit Speed Value $(\mathrm{v} \approx 1)$

In this phase, predators and prey will be in the same position. They have identical velocity. In this phase, there will be a shift from the character of exploration to exploitation. The composition between the prey as exploration and predators as exploitation will be in the same number.

- For the first half of the population

$$
\begin{aligned}
& \overrightarrow{S_{l}}=\overrightarrow{X_{L}} \otimes\left({\overrightarrow{\text { Elte }_{i}}}_{-} \overrightarrow{X_{L}} \otimes \overrightarrow{\text { Prey }_{i}}\right) \quad i=1,2 \ldots . n / 2 \\
& {\overrightarrow{\text { Prey }_{i}}}={\overrightarrow{\text { Prey }_{i}}}_{C} \times \overrightarrow{\text { rand }} \otimes \overrightarrow{S_{l}}
\end{aligned}
$$

- For the second half of the population

$$
\begin{aligned}
& \overrightarrow{S_{l}}=\overrightarrow{X_{b}} \otimes\left(\overrightarrow{X_{b}} \otimes{\overrightarrow{\text { llte }_{i}}}-\overrightarrow{\text { Prey }}_{i}\right) i=n / 2, \ldots . n \\
& {\overrightarrow{\text { Prey }_{i}}}={\overrightarrow{\text { Prey }_{i}}}+C \times A c \otimes \overrightarrow{S_{l}}
\end{aligned}
$$




$$
A c=\left(1-\frac{\text { Iter }}{\text { Max_iter }}\right)^{\left(2 \frac{\text { Iter }}{\text { Max_iter }}\right)}
$$

where $\overrightarrow{R_{L}}$ is a random vector shaped from the levy allocation which depicts the levy motion. $A c$ is an adaptive controller that is employed to maintain the steps of predator action.

Phase 3: In Low-Speed Value ( $v=0.1)$

The last phase is synonymous with high exploitation. The predator will accelerate faster than the prey.

$$
\begin{aligned}
& \overrightarrow{S s_{l}}=\overrightarrow{X_{L}} \otimes\left(\overrightarrow{X_{L}} \otimes{\overrightarrow{\text { Ellte }_{i}}}_{-} \otimes \overrightarrow{\text { Prey }}_{i}\right) i=1 \ldots n \\
& {\overrightarrow{\text { Prey }_{i}}}={\overrightarrow{\text { Prey }_{i}}}+C \times A c \otimes{\overrightarrow{S s_{l}}}
\end{aligned}
$$

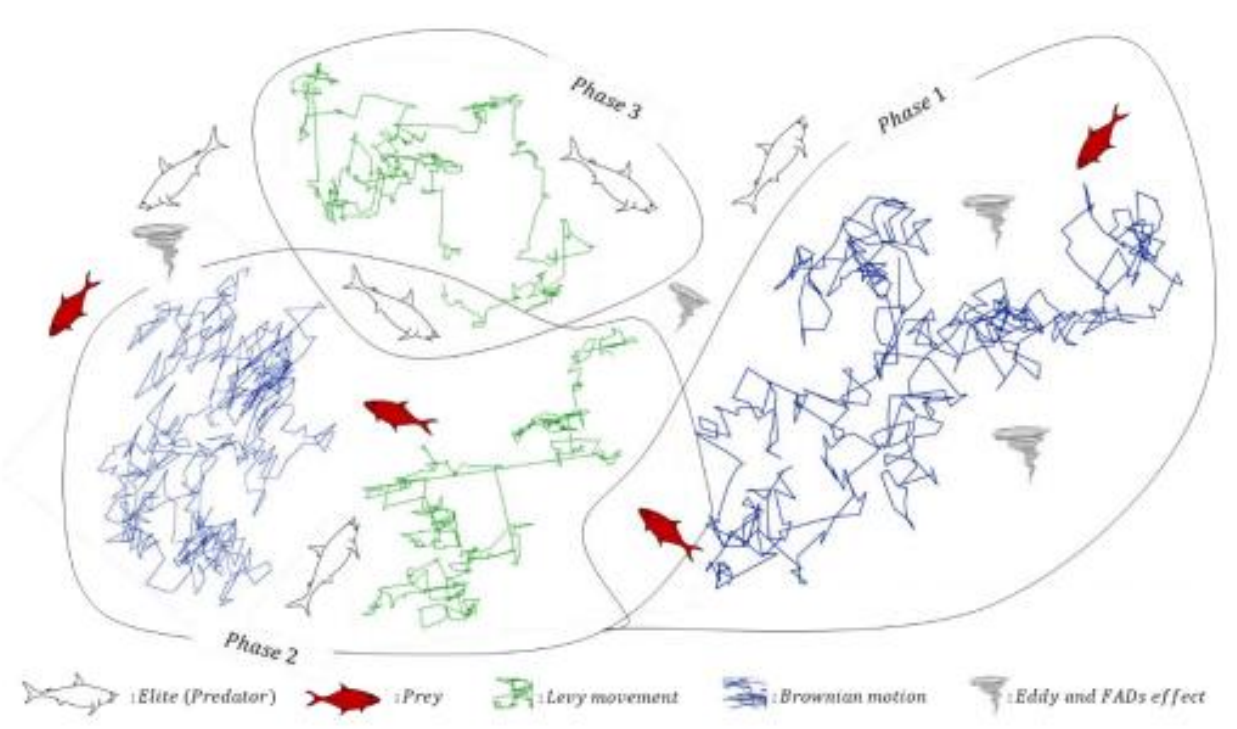

Figure 2. The Three MPA Optimization Phases [19]

\subsection{A Sine Tree-Seed Algorithm (STSA)}

The STSA method is to improve the ability of the Tree-Seed Algorithm (TSA) based on the inspiration of the Sine Cosine algorithm (SCA). TSA has weaknesses in optimizing multimodal and high-order objective functions [20]. Seeds have an important role in the distribution and search for optimal value. This is not optimal due to random and simple seed production. Poor seed production will result in optimization results that are not in accordance with the optimal solution. The STSA method modifies the the number of seeds (ns) value so that it can be processed according to changes in the function evaluations (FE) value. This has an effect on the amount that impacts on the best solution finding model. Population-based concept in STSA using trees and seeds which can be formulated as follows:

$$
\begin{aligned}
& T_{i, j}^{t+1}=T_{j, \text { min }}^{t}+r_{i, j}^{t} \times\left(U_{j, \text { max }}^{t}-U_{j, \text { min }}^{t}\right) \\
& S_{i, j}^{t+1}=T_{i, j}^{t}+\alpha_{i, j}^{t} \times\left(S_{j, \text { max }}^{t}-T_{r, j}^{t}\right) \\
& S_{i, j}^{t+1}=T_{i, j}^{t+1}+\beta_{i, j}^{t} \times\left(T_{i, j}^{t}-T_{r, j}^{t}\right) \\
& \left.S_{j, \text { max }}^{t}=\min \left(\int_{\left(T_{l, j}^{t+1}\right.}\right)\right)
\end{aligned}
$$

where the vector of the tree is $T_{i, j}^{t+1}$. The higher bound of the search space is $U_{j, \max }^{t}$. The lower bound of the search space is $U_{j, \text { min }}^{t}$. The random value with range $[0,1]$ generated for each dimension is $r_{i, j}^{t}$. The 
vector of the seed is $S_{i, j}^{t+1} \cdot \beta_{i, j}^{t}$ are the scaling element which generated in series of $[-1,1]$ randomly. The vector of top tree area is $S_{j, \max }^{t}$. The vector of a tree randomly selected is $T_{r, j}^{t}$.

\subsection{Chimp Optimization Algorithm (ChOA)}

The Chimp Optimization Algorithm (ChOA) is imbued by the individual ingenuity and sexual motivation of chimpanzees in group hunting. This is different from other social predators. ChOA is modeled to further ease the two problems of slow convergence speed and trapped local optima in solving high dimensional problems. Chimpanzee colonies have four kinds of tasks, namely driver, barrier, hunter, and attacker. This is to push the hunt. The driver has a duty to monitor his prey without chasing it. The barrier is an obstacle to the motion of the prey by setting up a barrier. The chaser will pursue the prey without attack. The attacker is to forecast the fleeing way of the prey in anear area.The appropriate ChOA specifications are established [21]. The ChOA algorithm has four main stages, namely:

- Driving and chasing the prey

During the exploration and exploitation stages, prey is hunted. The driver and chaser mathematical equation can be modeled as follows:

$$
\begin{aligned}
& x=\left|y \cdot s_{\text {prey }}-m \cdot s_{\text {chimp }}\right| \\
& s_{\text {chimp }}(t+1)=s_{\text {prey }}(t)-z \cdot x
\end{aligned}
$$

where the number of current iteration is $t$. The coefficient vectors are $\mathrm{x}, y$, and $z$. The vector of prey position is $s_{\text {prey. }}$. The position vector of a chimp is $s_{\text {chimp }}$.

- Exploration phase

Chimpanzee movements in attack can be described in 2 models, namely: 1. the chimpanzee will explore the prey area (driver, barrier, and chaser), and 2. the chimp will surround the prey area. lastly, the attacker will condition the hunt. At the start of the iteration, the prey position and the chaser position are assumed to be the same. On the other hand, the position of the driver, barrier, and chaser will be set with reference to the position of the attacker. the best position will be a reference in updating the position of the chimpanzee.

- Exploitation phase In this phase, the chimps will stop hunting when the prey stops moving. The chimps will start attacking the prey. New chimpanzee position between current position and prey position.

- Improved the exploitation phase using the social incentive (sexual motivation)

The movement exploration and exploitation of chimpanze can be ilustrated in Figure 3.

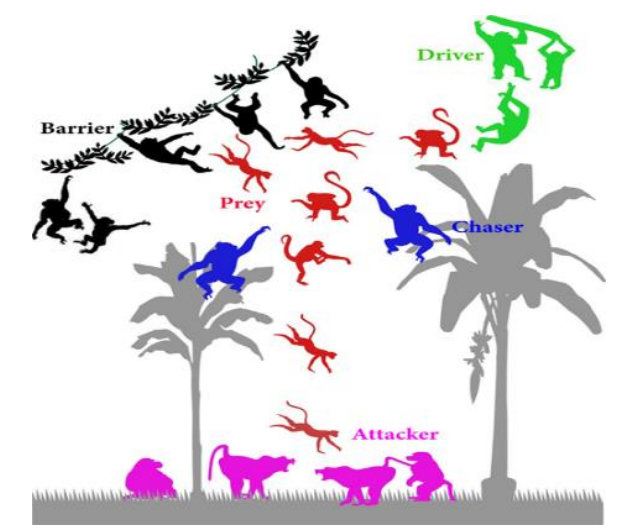

a)

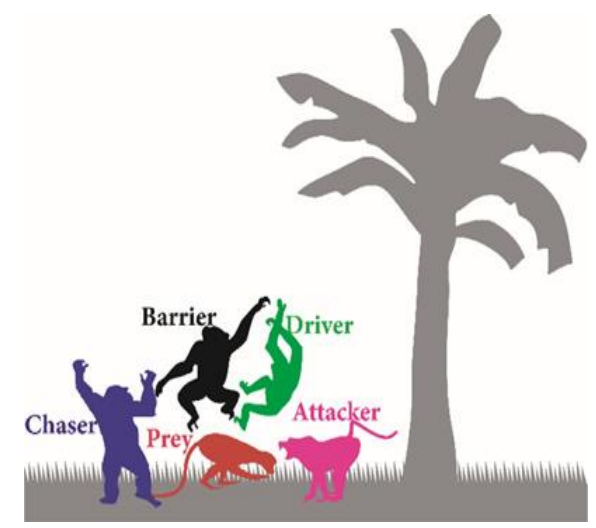

b)

Figure 3. The Basic Movement of Chimpanzee Colonies a) Exploration phase and b) Exploitation phase [21] 


\subsection{Equilibrium Optimizer (EO)}

The Equilibrium Optimizer (EO) metaheuristic method is inspired by the volume-control mass stability design taken to assess dynamic and equilibrium conditions. Each object with its concentration is to serve as a search agent. randomly, the search agent updates the concentration with respect to the best solution, namely the candidate equilibrium. it aims to achieve a state of equilibrium (optimal results) [22].

There are three phases presented for updating a particle. It is operated independently. The first phase is referred to as the equilibrium concentration. Selection of the best solution runs randomly based on groups. This group is called the equilibrium group. The second phase serves as a direct search mechanism. It is based on the difference in concentration between the particles and the equilibrium state. Particles act as explorers to search for regions globally. The third phase is called the generation rate phase. In this phase, the particles mostly serve as exploiters or pure solutions. However, sometimes the particles act as explorers as well. At the beginning of the iteration, the population based on the number of particles and dimensions is randomly and uniformly selected in the search space. Mathematical equations can be formulated as follows:

$$
P_{i}^{\text {initial }}=P_{\min }+\operatorname{rand}\left(P_{\max }-P_{\min }\right) \quad i=1,2, \ldots n
$$

where the initial concentration vector of the ith particle is $P_{i}^{\text {initial }}$. The minimum and maximum rates for the dimensions are $P_{\min }$ And $P_{\max }$. A random vector in the interval $[0,1]$ is denoted by rand. the number of particles as a population is represented by $n$.

\subsection{Giza Pyramids Construction (GPC)}

Giza Pyramids Construction (GPC) is inspired by the ancient past and features a good metaheuristic algorithm to handle many problems. The inspiration of antiquity is to observe and reflect on the legacy of the past in order to understand the optimal methods, technologies, and strategies of that era[23]. The proposed algorithm is controlled by the movement of workers and pushing stone blocks on the road.

Workers have to arrange the scattered stone blocks and push them into the place of installation. Ramps are used to move blocks of stone by taking into account slope and friction. The workers are tried to find the best position to move the stone blocks. The workers will always be updated to balance the ability of workers to move stone blocks. There are some GPC rules as follows:

1. The pyramids is build using a straight-on ramp

2. Suppose that only one ramp is used.

3. Ramp angle with a horizon of less than $15^{\circ}$ and may vary.

4. The solution comes from the resultant position of the worker and the stone block. Because the worker was actually pushing a stone block.

5. Friction is taken into account in stone block displacement but not considered for workers.

6. During the construction process, workers are renewed and placed in new area

\subsection{Mathematical Benchmark Functions}

The first step is to consider 23 literary mathematical functions. The mathematical function consists of 7 unimodal F1-F7, 6 Multimodal F8-F13, and 10 fixed-dimensional multimodal functions F14-F23. The functions are presented in Figure 4. The number of iterations and the population used are 25 and 50, respectively. 


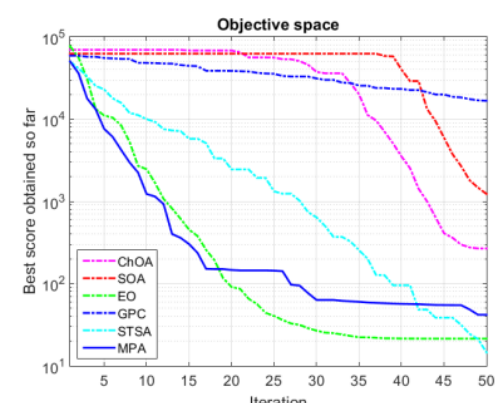

a)

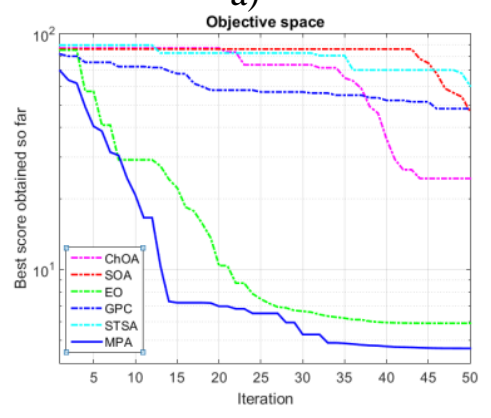

d)

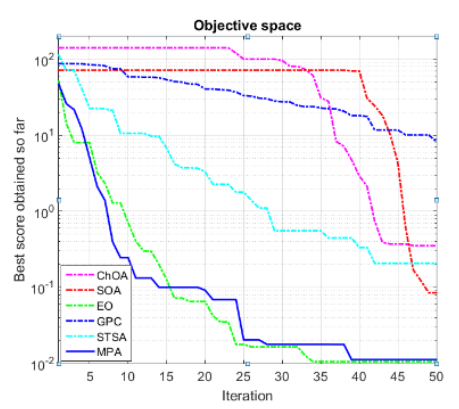

g)

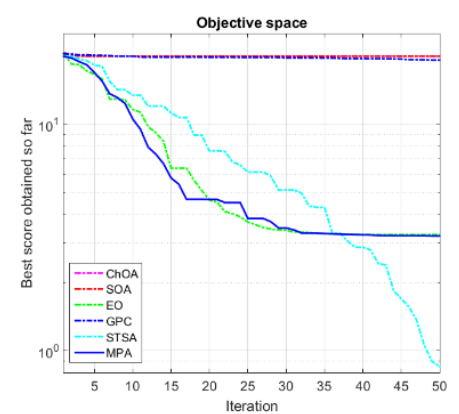

j)

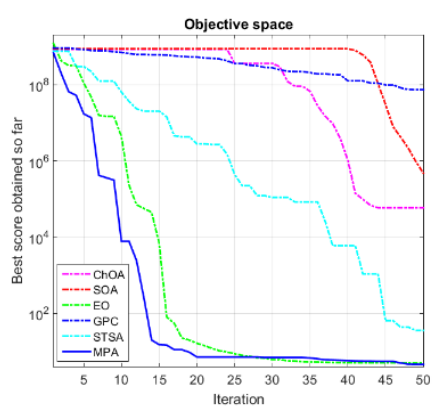

m)

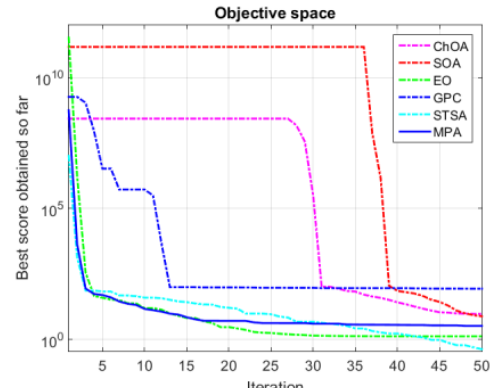

b)

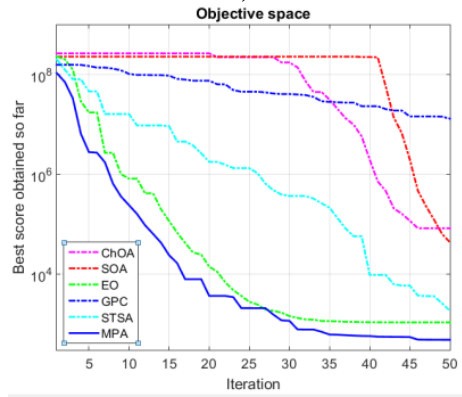

e)

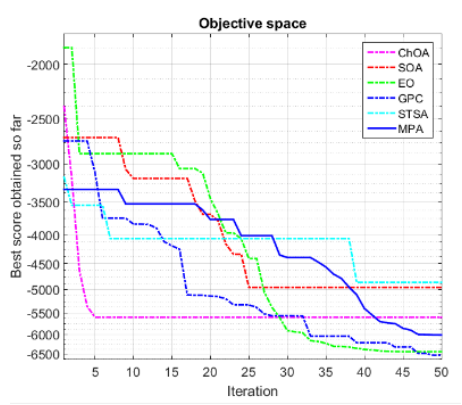

h)

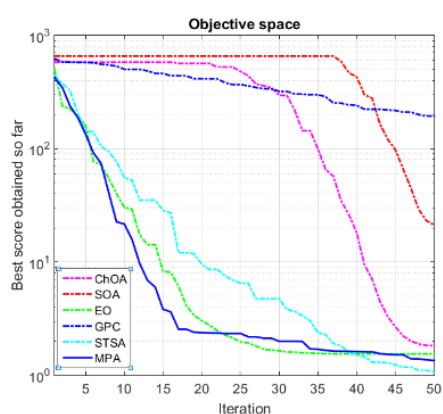

k)

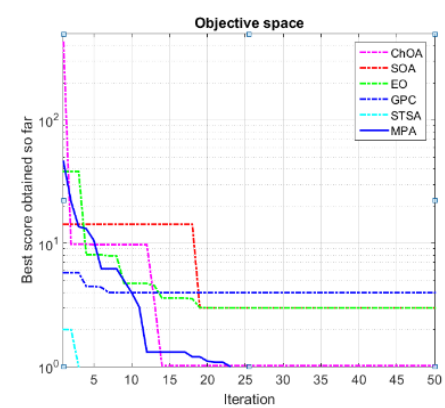

n)

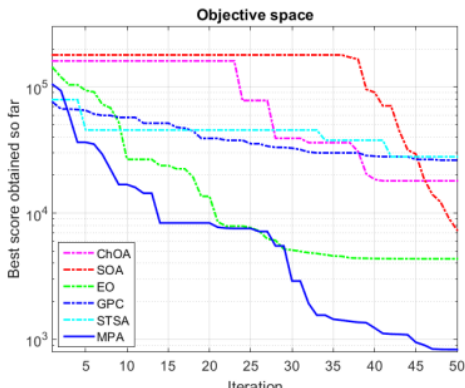

c)

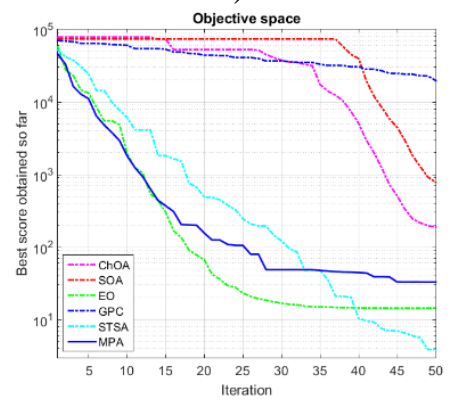

f)

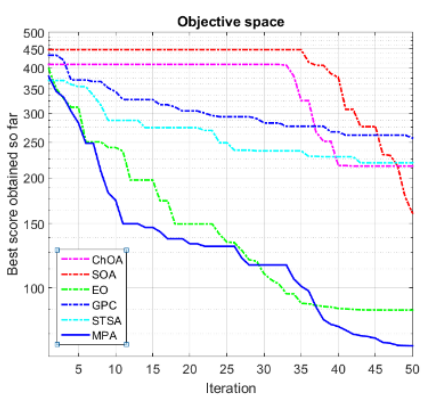

i)

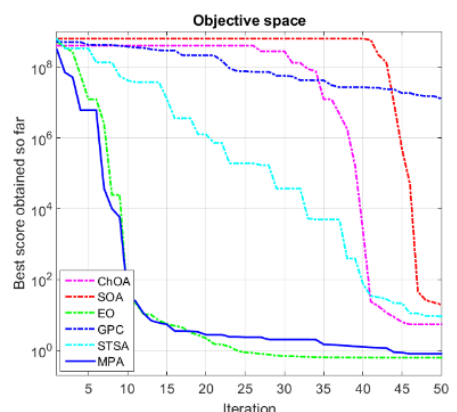

1)

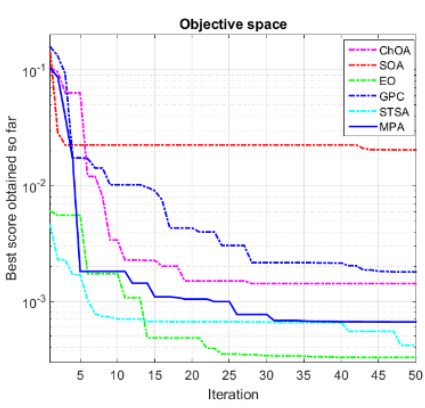

o) 


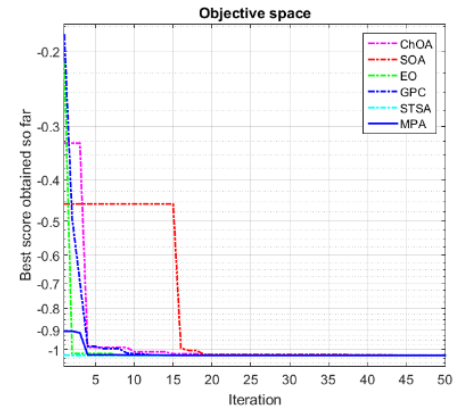

p)

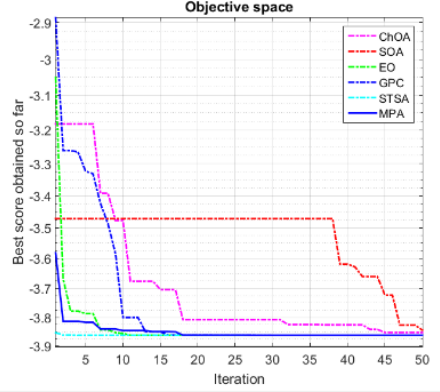

s)

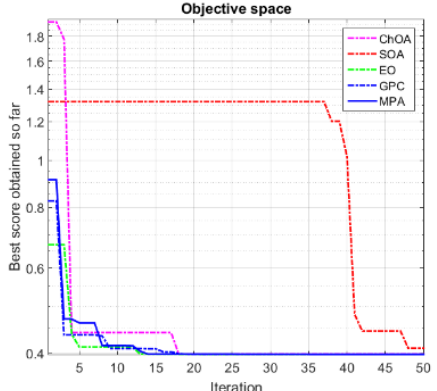

q)

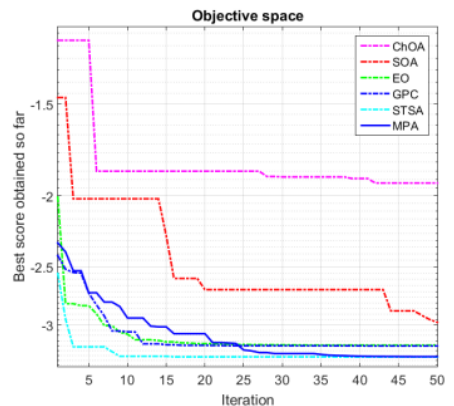

t)

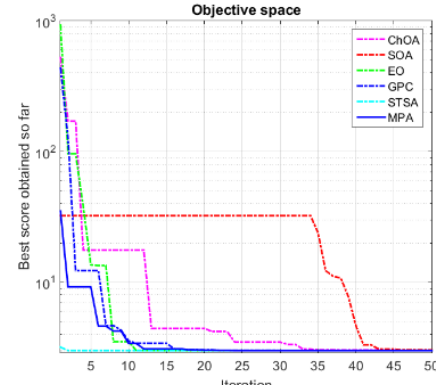

r)

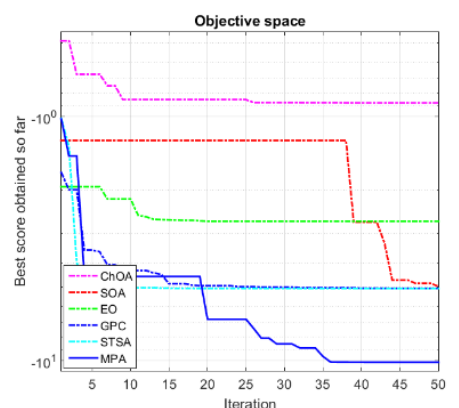

u)

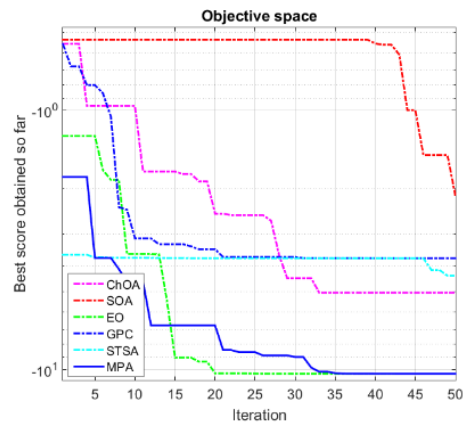

v)

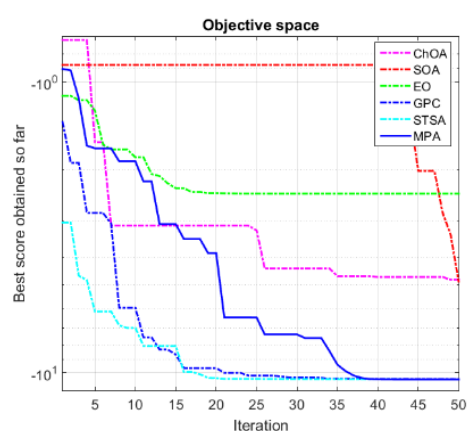

W)

Figure 4. The Convergence Curve of Benchmark Function Comparison a) F1, b) F2, c) F3, d) F4, e) F5, f) F6, g) F7, h) F8, i) F9, j) F10, k) F11, l) F12, m) F13, n) F14, o) F15, p) F16, q) F17, r) $F 18$, s) F19, t) F20, u) F21, v) F22 and w) F23

\section{RESULTS AND DISCUSSION}

Several studies have been carried out using metaheuristic methods and numerical programming methods to get the best solution from ELD with various constraints. The metaheuristic method presented will be verified for its feasibility and robustness in solving ELD problems. it uses three case studies with different characteristics. It is using a population size of 50 to determine performance and distinguish variations during the iteration process and convergence characters. Next, we present a comparison between the solution quality and computational efficiency of this method with performance other methods. The test of the metaheuristic method on ELD used 2 case studies, namely case 1 with 3 power systems and case 2 with 6 power systems. Power system data are derived from reference literature.

\subsection{Case Study with 3 Power Systems}

The first study is used three power system units to determine the performance of the metaheuristic method to determine the best power plant in this power system. In this study, load requirements data (PD) $150 \mathrm{MW}$ are used. More complete characteristics of the cost coefficient and generating capacity taking into account the transmission loss coefficient can be seen in Table 1. It is a system with 3 thermal power systems known as the P1, P2, and P3 generators. The scheme of 3 generators with 5 buses can be seen in Figure 5 


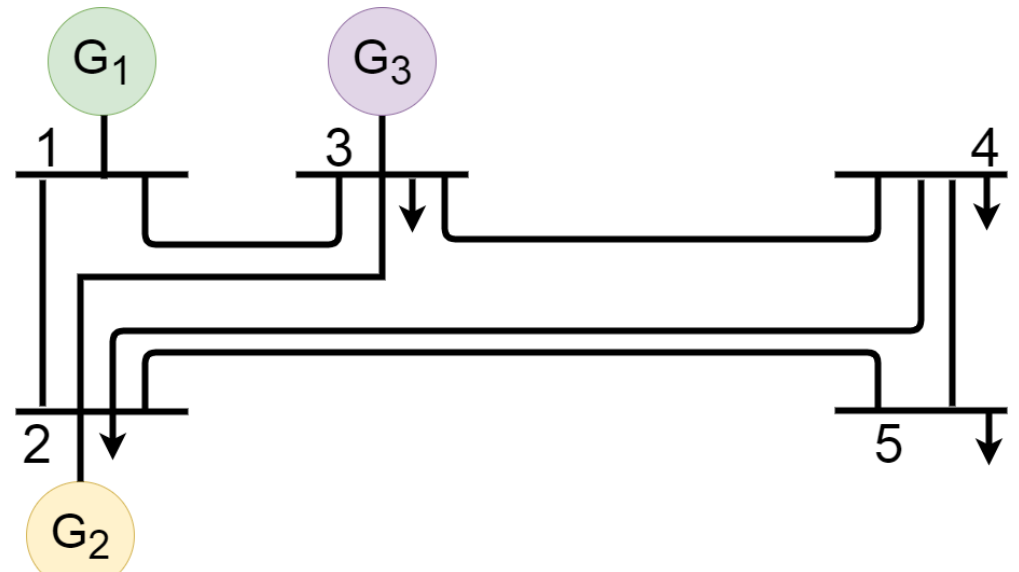

Figure 5. Single Line Diagram of The Electric Power System 3 Generators - 5 Buses [25]

Table 1. Power system data 3 units

\begin{tabular}{|l|l|l|l|l|l|}
\hline $\mathrm{P}_{1}(\mathrm{MW})$ & $\alpha_{i}(\$ / \mathrm{h})$ & $\beta_{i}(\$ / \mathrm{MW} \mathrm{h})$ & $\Upsilon_{i}\left(\$ / \mathrm{MW}^{2} \mathrm{~h}\right)$ & $\mathrm{P}_{\min }(\mathrm{MW})$ & $\mathrm{P}_{\max }(\mathrm{MW})$ \\
\hline $\mathrm{P}_{1}$ & 0.008 & 7.00 & 200 & 10 & 85 \\
\hline $\mathrm{P}_{2}$ & 0.009 & 6.30 & 180 & 10 & 80 \\
\hline $\mathrm{P}_{3}$ & 0.007 & 6.80 & 140 & 10 & 70 \\
\hline
\end{tabular}

Loss coefficient matrix B is used to obtain transmission line losses and meet transmission capacity limits. Software code written in Matlab software is using a computer with an AMD A6 processor with a RAM capacity of $4 \mathrm{~GB}$.

$$
B=\left[\begin{array}{lll}
0.000218 & 0.000093 & 0.000028 \\
0.000093 & 0.000228 & 0.000017 \\
0.000028 & 0.000017 & 0.000179
\end{array}\right]
$$

In Table 2, it can be seen the value of the estimated load on each generator of the 3 power systems, the total power generated, costs, and power losses. In Table 2, the STSA method cost is $1597.48152 \$ / \mathrm{h}$. The value is best compared to other mathematical and metaheuristic methods. The total value of the loss at this optimal dispatch using the STSA method is 152.3419 MW. Figure 6 is a graph of the comparison of the loss transmission at the optimal dispatch. The STSA method has the same the loss at this optimal dispatch as the mathematical method.

Table 2. Forecast output power for 3 Power System with PD $=150 \mathrm{MW}$

\begin{tabular}{|l|l|l|l|l|l|l|l|}
\hline $\mathrm{P}_{1}(\mathrm{MW})$ & $\begin{array}{c}\text { Math } \\
\text { method }\end{array}$ & ChOA & SOA & EO & GPC & STSA & MPA \\
\hline $\mathrm{P}_{1}$ & 33.4701 & 32.576939 & 32.445926 & 32.810106 & 32.821883 & 32.817346 & 32.810133 \\
\hline $\mathrm{P}_{2}$ & 64.0974 & 64.733446 & 65.335612 & 64.595012 & 64.589653 & 64.582312 & 64.595079 \\
\hline $\mathrm{P}_{3}$ & 55.1011 & 55.032253 & 54.568919 & 54.936924 & 54.930498 & 54.942246 & 54.93683 \\
\hline PL (MW) & 2.3419 & 2.3426382 & 2.3504572 & 2.342041 & 2.3420344 & 2.3419047 & 2.3420421 \\
\hline $\mathrm{Pi}(\mathrm{MW})$ & 152.3419 & 152.35239 & 152.35046 & 152.34204 & 152.34203 & 152.3419 & 152.34204 \\
\hline PD MW) & 150 & 150 & 150 & 150 & 150 & 150 & 150 \\
\hline Cost (\$/h) & 1599.98 & 1597.4823 & 1597.4894 & 1597.48152 & 1597.48152 & 1597.48152 & 1597.48152 \\
\hline
\end{tabular}




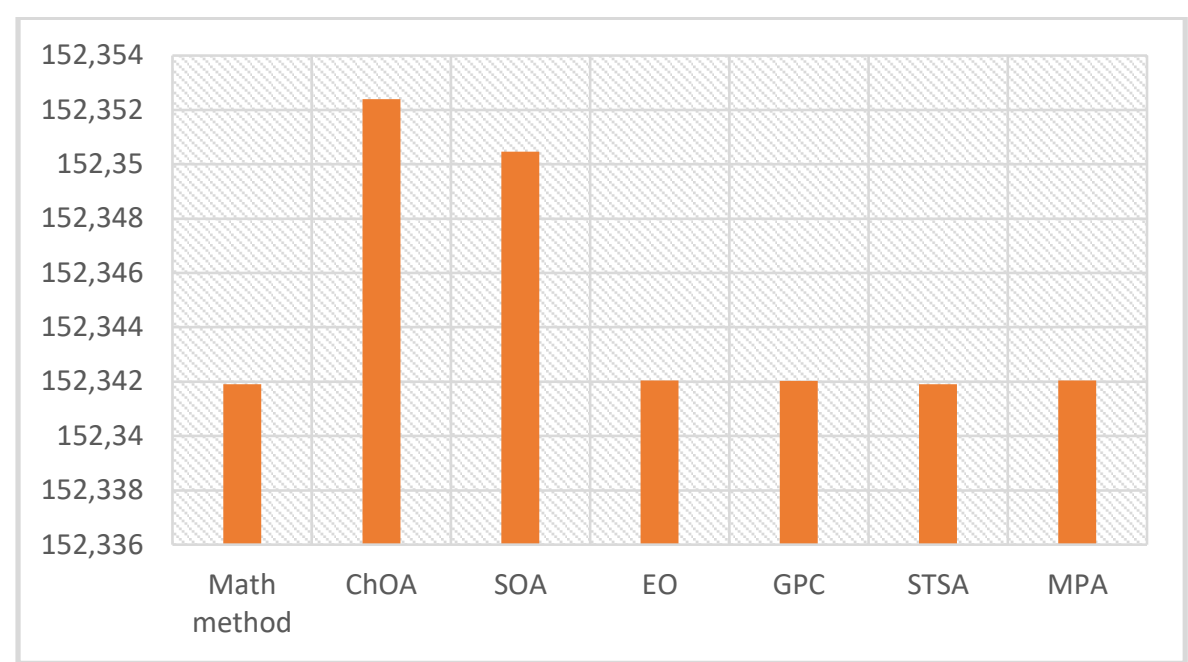

Figure 6. Comparison Chart of The Loss Transmission (3 Unit Power System)

In Table 3, it can be seen the comparison of each metaheuristic method tested on 3 power systems with a power demand of $150 \mathrm{MW}$ by entering the calculation of transmission line losses. The report is displayed by entering the worst cost, mean cost, best cost and standard deviation (STD).

Table 3. Comparison of The Cost In 3 Power Systems $(P D=150 \mathrm{MW})$

\begin{tabular}{|l|c|c|c|c|}
\hline \multicolumn{1}{|c|}{$\mathrm{P}_{1}(\mathrm{MW})$} & Worst cost $(\$ / \mathrm{h})$ & Mean cost $(\$ / \mathrm{h})$ & Best cost $(\$ / \mathrm{h})$ & $\mathrm{STD}(\$ / \mathrm{h})$ \\
\hline ChOA & 1602.717 & 1597.8107 & 1597.4823 & 1.1282 \\
\hline SOA & 1608.1434 & 1598.7501 & 1597.4894 & 2.2099 \\
\hline EO & 1597.48152 & 1597.48152 & 1597.48152 & 0 \\
\hline GPC & 1598.2839 & 1597.5364 & 1597.48152 & 0.1932 \\
\hline STSA & 1597.632 & 1597.4991 & 1597.48152 & 0.0379 \\
\hline MPA & 1600.8281 & 1597.8146 & 1597.48152 & 0.8875 \\
\hline
\end{tabular}

\subsection{Case Study with 6 Power Systems}

The second test is using 6 units of the power system. This is to obtain an effective comparison between the metaheuristic methods used in this paper. This is to get an estimate of the power load. The 6 unit thermal type power system design data is shown in Table 4. Meanwhile, the transmission loss coefficient matrix is represented by $B$. In this second test, PD of $1263 \mathrm{MW}$ is used. In Figure 7, It can be seen a single line diagram of 6 generators with 26 buses.

Table 4. Power System Data 6 Units

\begin{tabular}{|l|c|c|c|c|c|}
\hline $\mathrm{P}_{1}(\mathrm{MW})$ & $\alpha_{i}(\$ / \mathrm{h})$ & $\beta_{i}(\$ / \mathrm{MW} \mathrm{h})$ & $\Upsilon_{i}\left(\$ / \mathrm{MW}^{2} \mathrm{~h}\right)$ & $\mathrm{P}_{\min }(\mathrm{MW})$ & $\mathrm{P}_{\max }(\mathrm{MW})$ \\
\hline $\mathrm{P}_{1}$ & 0.007 & 7.00 & 240 & 100 & 500 \\
\hline $\mathrm{P}_{2}$ & 0.0095 & 10 & 200 & 50 & 200 \\
\hline $\mathrm{P}_{3}$ & 0.009 & 8.5 & 220 & 80 & 300 \\
\hline $\mathrm{P}_{4}$ & 0.009 & 11 & 200 & 50 & 150 \\
\hline $\mathrm{P}_{5}$ & 0.008 & 10.5 & 220 & 50 & 200 \\
\hline $\mathrm{P}_{6}$ & 0.0075 & 12 & 190 & 50 & 120 \\
\hline
\end{tabular}




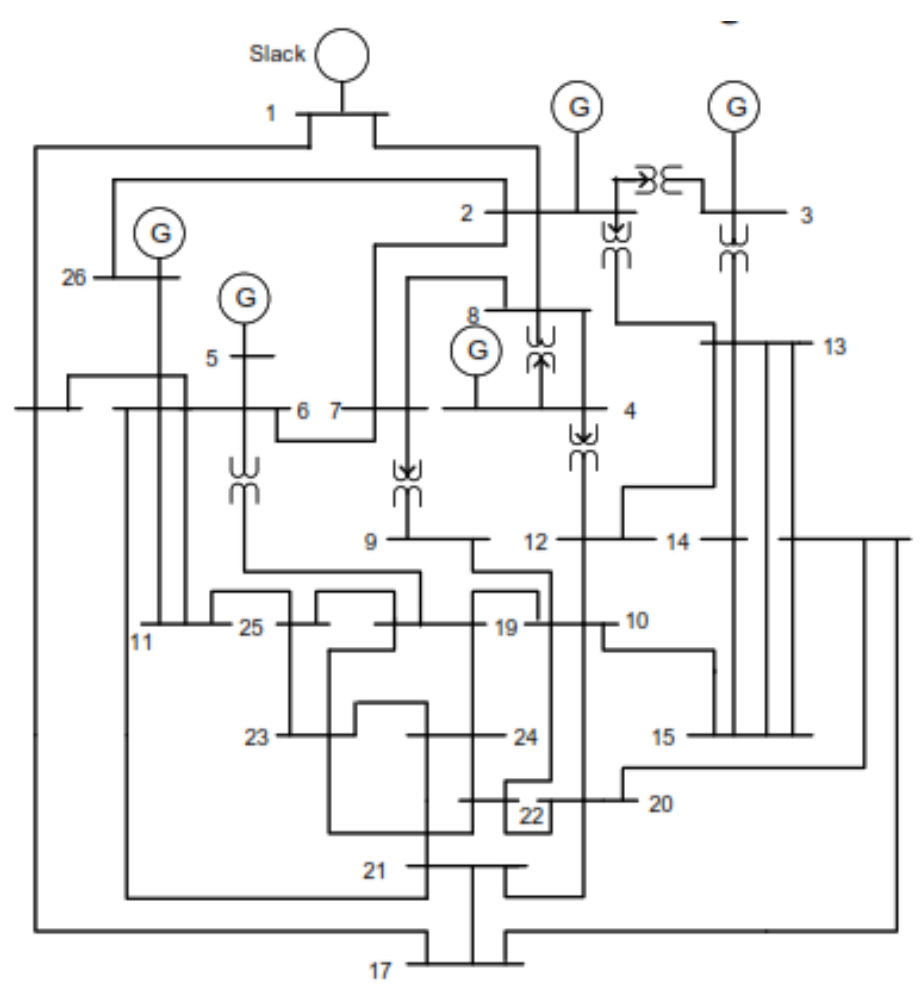

Figure 7. Single Line Diagram of The Electric Power System 6 Generators - 26 Buses

$$
B=10^{-3} \times\left[\begin{array}{rccccc}
0.017 & 0.012 & 0.007 & -0.001 & -0.005 & -0.002 \\
0.012 & 0.014 & 0.009 & 0.001 & -0.006 & -0.001 \\
0.007 & 0.009 & 0.031 & 0 & -0.01 & -0.006 \\
-0.001 & 0.001 & 0 & 0.024 & -0.006 & -0.008 \\
-0.005 & -0.006 & -0.010-0.006 & 0.129 & -0.002 \\
-0.002 & -0.001 & -0.0060-0.008 & -0.02 & 0.15
\end{array}\right]
$$

In Table 5, it can be seen the results of the estimated load and cost of generating each power system unit in the 6 case study of the power system. Table 5 shows the power loss, total power and cost. The estimation results using the STSA method obtained the best results compared to other metaheuristic methods in this paper. The value is $15444.0226(\$ / \mathrm{h})$. The total value of the loss transmission at the optimal dispatch using the STSA method is 1275.2029 MW. Figure 8 is a graph of the comparison of losses in case 2 . The result that is close to the value of the STSA method is the EO method.

Table 5. Forecast Output Power For 6 Power System with PD $=1263$ MW

\begin{tabular}{|c|c|c|c|c|c|c|}
\hline Generator & ChOA & SOA & EO & GPC & STSA & MPA \\
\hline $\mathrm{P}_{1}$ & 430.6253426 & 430.3783077 & 438.6744125 & 416.6701414 & 444.6499234 & 433.3745579 \\
\hline $\mathrm{P}_{2}$ & 166.3819825 & 200 & 200 & 200 & 171.7126716 & 163.0587002 \\
\hline $\mathrm{P}_{3}$ & 254.206883 & 246.6733607 & 257.6396762 & 300 & 261.1550089 & 253.5746587 \\
\hline $\mathrm{P}_{4}$ & 150 & 123.552395 & 150 & 150 & 150 & 150 \\
\hline $\mathrm{P}_{5}$ & 200 & 155.0460548 & 159.8288332 & 143.668211 & 162.7314736 & 200 \\
\hline $\mathrm{P}_{6}$ & 74.54014127 & 120 & 69.07272306 & 64.93809964 & 84.95378805 & 75.74745118 \\
\hline $\mathrm{PL}(\mathrm{MW})$ & 12.754349 & 12.650118 & 12.215645 & 12.276452 & 12.202865 & 12.755368 \\
\hline $\mathrm{Pi}(\mathrm{MW})$ & 1275.7543 & 1275.6501 & 1275.2156 & 1275.2765 & 1275.2029 & 1275.7553 \\
\hline $\mathrm{PD}(\mathrm{MW})$ & 1263 & 1263 & 1263 & 1263 & 1263 & 1263 \\
\hline Cost $(\$ / \mathrm{h})$ & 15460.2548 & 15468.3407 & 15454.82890 & 15479.01406 & 15444.0226 & 15460.0783 \\
\hline
\end{tabular}




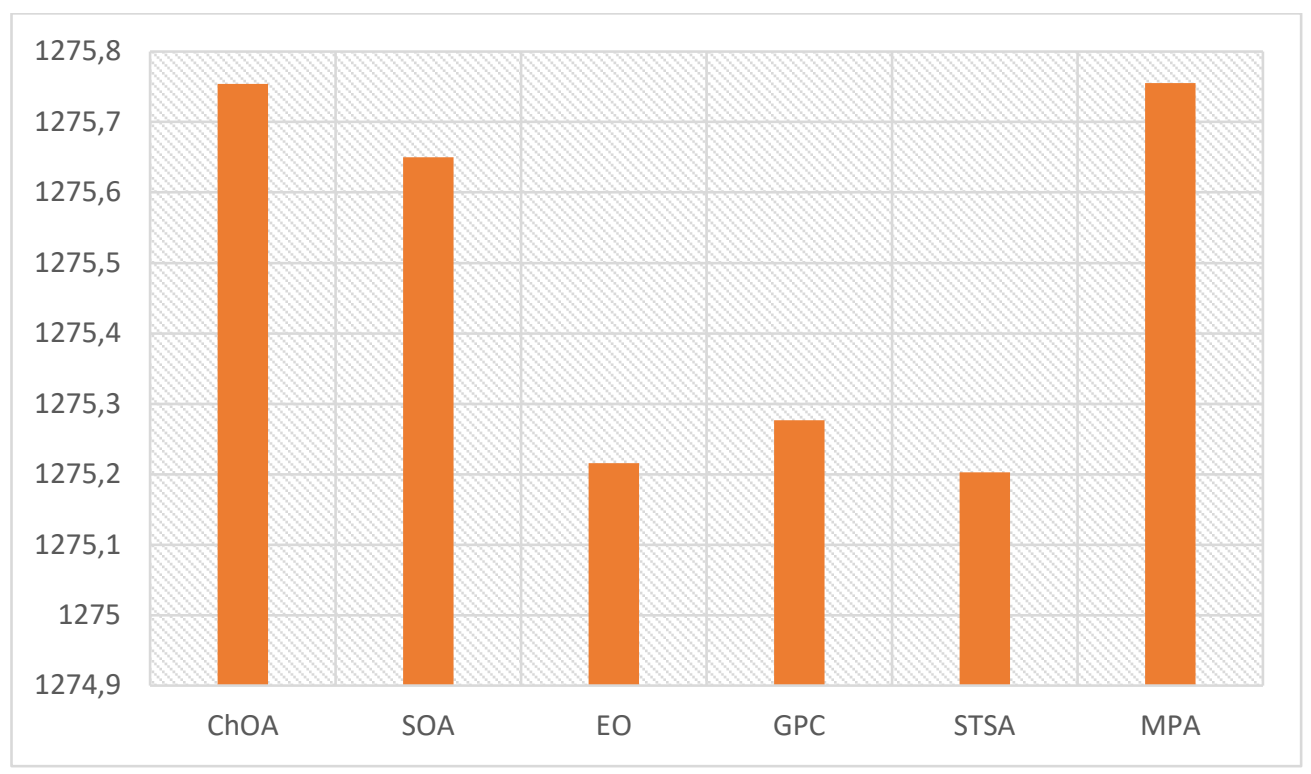

Figure 8. Comparison Chart of The Loss Transmission (6 Unit Power System)

Table 6 is the comparison of the worst, average, best and STD costs of the metaheuristic method in this paper by testing 6 units of the power system.

Table 6. Comparison of the Cost In 6 Power Systems $(P D=1263 \mathrm{MW})$

\begin{tabular}{|c|c|c|c|c|}
\hline $\mathrm{P}_{1}(\mathrm{MW})$ & Worst cost $(\$ / \mathrm{h})$ & Mean cost $(\$ / \mathrm{h})$ & Best cost $(\$ / \mathrm{h})$ & $\mathrm{STD}(\$ / \mathrm{h})$ \\
\hline ChOA & 15541.87073 & 15498.69812 & 15460.25488 & 35.10411333 \\
\hline SOA & 15627.27653 & 15546.86006 & 15468.34076 & 58.45088829 \\
\hline EO & 15499.24175 & 15471.17991 & 15454.82892 & 11.96734174 \\
\hline GPC & 15627.27653 & 15486.37974 & 15479.01406 & 29.79223595 \\
\hline STSA & 15491.42303 & 15451.98118 & 15444.02266 & 12.08607801 \\
\hline MPA & 15556.09063 & 15483.77228 & 15460.07834 & 26.54177549 \\
\hline
\end{tabular}

\section{CONCLUSION}

In this paper, an effective solution to the Economic Load Dispatch (ELP) problem has been explored by using 6 metaheuristic methods. These methods are Chimp Optimization Algorithm (ChOA), Seagull Optimization Algorithm (SOA), Equilibrium Optimizer (EO), Giza Pyramids Construction (GPC), A Sine Tree-Seed Algorithm (STSA) And Marine Predators Algorithm (MPA). In finding the best and effective solution, testing is used with 2 models, namely with 3 units and 6 units of power systems. From the experiment, it was found that the Sine Tree-Seed Algorithm (STSA) method has the best price compared to other methods in this paper. In test 1 , the cost value is $1597.48152 \$ / \mathrm{h}$. Meanwhile in test 2 , the cost value is $15444.0226 \$ / \mathrm{h}$.

\section{CONFLICTS OF INTEREST}

No conflict of interest was declared by the author. 


\section{REFERENCES}

[1] Aribowo, W., "Tuning for Power System Stabilizer Using Distributed Time-Delay Neural Network", SINERGI, 22(3): 205-210, (2017).

[2] Khan, I., Li, Z., Gu, W., Xu, Y., "Distributed control algorithm for optimal reactive power control in power grids", International Journal of Electrical Power and Energy Systems, 83: 505-513, (2016).

[3] Liang, H., Liu, Y., Shen, Y., Li, F., Man, Y., “A Hybrid Bat Algorithm for Economic Dispatch with Random Wind Power”, in IEEE Transactions on Power Systems, 33(5): 5052-5061, (2018).

[4] Priyanto, T. K., Maulana, M. F., Giyantara, A., "Dynamic economic dispatch using chaotic bat algorithm on $150 \mathrm{kV}$ Mahakam power system", 2017 International Seminar on Intelligent Technology and Its Applications (ISITIA), Surabaya, 116-121, (2017).

[5] Liang, H., Liu, Y., Shen, Y., Li, F., "A multiobjective chaotic bat algorithm for economic and emission dispatch", 2017 Chinese Automation Congress (CAC), Jinan, 4684-4689, (2017).

[6] Adarsh, B. R., Raghunathan, T., Jayabarathi, T., Yang, X. S., "Economic dispatch using chaotic bat algorithm", Energy, 96: 666-675, (2016).

[7] Rabiee, A., Jamadi, M., Mohammadi-Ivatloo, B., Ahmadian, A., "Optimal Non-Convex Combined Heat and Power Economic Dispatch via Improved Artificial Bee Colony Algorithm”, Processes, 8, (2020).

[8] Sharifi, S., Sedaghat, M., Farhadi, P., Ghadimi, N., Taheri, B., "Environmental economic dispatch using improved artificial bee colony algorithm", Evolving Systems, 8: 233-242, (2017).

[9] Rao, R. K., Srinivas, P., Divakar, M. S. M., Venkatesh, G. S. N. M., "Artificial bee colony optimization for multi objective economic load dispatch of a modern power system", 2016 International Conference on Electrical, Electronics, and Optimization Techniques (ICEEOT), Chennai, 4097-4100, (2016).

[10] Jayabarathi, T., Raghunathan, T., Adarsh, B. R., Suganthan, P. N., "Economic dispatch using hybrid grey wolf optimizer", Energy, 111: 630-641, (2016).

[11] Hardiansyah, H., "Grey Wolf Optimizer Applied to Dynamic Economic Dispatch Incorporating Wind Power", Global Journal of Research in Engineering, 20(4-F), (2020).

[12] Mostafa, E., Abdel-Nasser, M., Mahmoud, K., "Application of mutation operators to grey wolf optimizer for solving emission-economic dispatch problem", 2018 International Conference on Innovative Trends in Computer Engineering (ITCE), Aswan, 278-282, (2018).

[13] Pradhan, M., Roy, P. K., Pal, T., "Oppositional based grey wolf optimization algorithm for economic dispatch problem of power system", Ain Shams Engineering Journal, 9(4): 2015-2025, (2018).

[14] Zhao, J., Liu, S., Zhou, M., Guo, X., Qi, L., "Modified cuckoo search algorithm to solve economic power dispatch optimization problems", in IEEE/CAA Journal of Automatica Sinica, 5(4): 794806, (2018).

[15] Nguyen, T.T., Nguyen, T.T., Vo, D. N., "An effective cuckoo search algorithm for large-scale combined heat and power economic dispatch problem", Neural Computing and Applications, 30: 3545-3564, (2018). 
[16] Nguyen, T. T., Vo, D. N., Dinh, B. H., "Cuckoo search algorithm for combined heat and power economic dispatch", International Journal of Electrical Power \& Energy Systems, 81: 204-214, (2016).

[17] Chellappan, R., Kavitha, D., "Economic and emission load dispatch using Cuckoo search algorithm", 2017 Innovations in Power and Advanced Computing Technologies (i-PACT), Vellore, 1-7, (2017).

[18] Dhiman, G., Kumar, V., "Seagull optimization algorithm: Theory and its applications for largescale industrial engineering problems", Knowledge-Based Systems, 165: 169-196, (2019).

[19] Faramarzi, A., Heidarinejad, M., Mirjalili, S., Gandomi, A. H., "Marine Predators Algorithm: A nature-inspired metaheuristic", Expert Systems with Applications, 152, (2020).

[20] Jiang, J., Xu, M., Meng, X., Li, K., "STSA: A sine Tree-Seed Algorithm for complex continuous optimization problems", Physica A: Statistical Mechanics and its Applications, 537, (2020).

[21] Khishe, M., Mosavi, M.R., “Chimp optimization algorithm”, Expert Systems with Applications, $149,(2020)$.

[22] Faramarzi, A., Heidarinejad, M., Stephens, B., Mirjalili, S., "Equilibrium optimizer: A novel optimization algorithm", Knowledge-Based Systems, 191, (2020).

[23] Harifi, S., Mohammadzadeh, J., Khalilian, M., Ebrahimnejad, S., "Giza Pyramids Construction: an ancient-inspired metaheuristic algorithm for optimization", Evolutionary Intelligence, (2020).

[24] Wood, A. J., Wollenberg, B. F., Power generation operation and control, 2nd edition, (2010).

[25] Saadat, H., Power system analysis, McGraw-Hill Companies, (2008). 